gend Optimismus, dass durch die Veränderungen tatsächlich neue Impulse geschaffen werden können. Insbesondere der letzte Teil der Veranstaltung hat einen wichtigen Dialog zwischen der mittleren Ebene und den Vertretern des Landes und der Fachpolitiken eingeleitet, der fortsetzungswürdig erscheint. Es wurde klar, dass die Veränderung der Verwaltungsorganisation in Nordrhein-Westfalen den Anfang einer Entwicklung darstellt. Aus den Regionalräten wird eine weitergehende Stärkung der regionalen Ebene, insbesondere durch die Direktwahl der Mitglieder, die Befugnis zur verbindlichen Entscheidungsfindung und die Ausstattung mit eigenen Finanzmitteln, gefordert. Diesem Zuwachs an Kompetenzen und politischer Legitimation wird von Seiten des Landes wie der Kommunen mit Skepsis begegnet. Eine weitere Stärkung der Regionalräte wird daher zumindest voraussetzen, dass diese sich in der nahen Zukunft bewähren und wirklich neue Impulse schaffen, Da aus verschiedenen
Lagern Skepsis und Kritik an dem Zuschnitt der Regionen laut wurde, wird in diesem Prozess auch darüber nachzudenken sein, ob die zukünftige Zusammenarbeit analog der Verwaltungsgrenzen der Regierungsbezirke beibehalten werden soll. Möglicherweise ermutigen die erhofften Impulse aber auch $\mathrm{zu}$ einer verstärkten themen- und interessenbezogenen regionalen Zusammenarbeit außerhalb der Institutionen, so dass sich die Frage des Regionszuschnitts erübrigt. Eine solche Zusammenarbeit wird sich aber erst einstellen, wenn es gelingt, einflussreiche Akteure der regionalen Ebene aus Politik, Wirtschaft und Verwaltung für die Arbeit der Regionalräte zu gewinnen.

\author{
Nikolaus Paffenholz \\ Zentralinstitut für Raumplanung \\ an der Universität Münster \\ Wilmergasse 12-13 \\ 48143 Münster
}

\title{
Zur Zukunft der Landesplanung
}

\author{
Neue Ansätze und Entwicklungen des Landesplanungsrechts
}

Die Aufgaben der Landesplanung befinden sich im Wandel. $\mathrm{Zu}$ der klassischen Anforderung, eine ordnungsgemäße und einheitliche Raumentwicklung zu gewährleisten, sind heute Ansprüche an Flexibilität und Kooperationsmöglichkeiten in den Planungsprozessen getreten. Dem soll auch die anstehende Novellierung des nordrhein-westfälischen Landesplanungsrechts Rechnung tragen. Dies nahm das Zentralinstitut für Raumplanung an der Universität Münster unter der Leitung seines Geschäftsführenden Direktors Prof. Dr. Hans D. Jarass, LL.M. zum aktuellen Anlass, sich auf seinem diesjährigen Symposium „Zur Zukunft der Landesplanung“ mit neuen Ansätzen und Entwicklungen des Landesplanungsrechts $\mathrm{zu}$ beschäftigen.* Die Veranstaltung fand am 29. Oktober 2001 in Münster unter der Beteiligung von Fachleuten aus Wissenschaft, Anwaltschaft sowie behördlicher und privater Planungspraxis statt.

Zunächst referierte Peter Wilhelm Schneider, Ltd. Ministerialrat in der Staatskanzlei des Landes Nordrhein-Westfalen, über „Neue Ansätze der Landespla- nung in Nordrhein-Westfalen“. Ausgangspunkt seiner Überlegungen war die Feststellung, dass das Land geordnet und überplant sei. Zudem hätten sich die Rahmenbedingungen für die Landesplanung durch das BauROG 1998 und europarechtliche Einwirkungen verändert. In Nordrhein-Westfalen käme die Umstrukturierung der Verwaltungsorganisation hinzu. Um diesen Anforderungen gerecht zu werden, stehe eine Novellierung des Landesplanungsrecht in verschiedenen Bereichen an. Das Verfahrensrecht könne vereinfacht werden, um schnellere Entscheidungen herbeizuführen und Rechtsunsicherheit zu vermeiden. Etwa solle den Regionalräten ein Initiativrecht gegenüber der Landesregierung zukommen. Außerdem sollten Gebietsentwicklungspläne künftig nur noch einem Anzeige- statt einem Genehmigungsverfahren unterworfen werden. Auch werde eine Straffung der landesplanerischen Vorgaben angestrebt, die durch eine Zusammenfassung der Instrumente des Landesentwicklungsprogramms und des Landesentwicklungsplans erreicht werden könnte. Als weiteres Ziel der Modernisierung nannte 
Schneider die Förderung der interkommunalen und regionalen Zusammenarbeit bei der Umsetzung der Landesplanung. Reibungsverluste könnten durch eine Optimierung der Kompetenzverteilung vermindert werden. Hierzu seien die kommunale Selbstverwaltung und die regionale Eigendynamik zu stärken. Das Ziel der Flexibilisierung von Planungsinstrumenten sei durch die verstärkte Anwendung von Vertrags- und Verhandlungslösungen zu erreichen. Auch seien Monitoring- und Controlling-Systeme zu implementieren, um die Reaktionsfähigkeit der Planungsträger zu verbessern. Besonderes Augenmerk richtete Schneider auf die Flächenpolitik und konstatierte, dass Flächenvermarktung und -bevorratung verbessert werden könnten. Auch bestünden Bestrebungen, die bau- und naturschutzrechtliche Eingriffs-/Ausgleichs-Problematik bereits auf Ebene der Gebietsentwicklungsplanung in Angriff zu nehmen. Eine Schlüsselrolle bei der Novellierung landesplanerischer Inhalte maß Schneider der gesellschaftlichen Mitwirkung bei. Die Landesregierung sehe daher die Gewährleistung eines diskursiven Planungsprozesses als eine ihrer wesentlichen Aufgaben bei der Novellierung an.

Die Regionalisierung in der Landesplanung korrespondiert mit Konzepten auf europäischer Ebene. Die "Raumentwicklung in Europa" stand somit im Mittelpunkt des zweiten Vortrags von Dr. Siegbert Gatawis, $L L . M$., ehemaligem Wissenschaftlichen Referenten am Zentralinstitut für Raumplanung. Unter den Instrumenten der Raumentwicklung in Europa stehe das "soft law", also rechtlich unverbindliche Vereinbarungen mit weitreichenden faktischen Auswirkungen, im Vordergrund. Zum raumwirksamen "soft law“ gehöre neben den Programmen "Europa 2000" und „Europa $2000+$ " vor allem das Europäische Raumentwicklungskonzept (EUREK). Das Ziel des Konzeptes bestehe in der Förderung sozialer, wirtschaftlicher und ökologischer Nachhaltigkeit. Durch das EUREK solle, so Gatawis, eine Bündelung und verbesserte Abstimmung der Fachpolitiken erreicht werden, wobei insbesondere die regionale und lokale Ebene sowie die grenzüberschreitende Zusammenarbeit gefördert werden sollten. Jedoch sei das EUREK für die Mitgliedstaaten nicht rechtsverbindlich. Es entfalte vielmehr eine mittelbare faktische Bindung durch Anreize in Form von Fördermitteln. Im deutschen Planungsrecht schlügen sich die Vereinbarungen des EUREK in verschiedenen Regelungen des Raumordnungsgesetzes nieder und untermauerten in der Landes- und Regionalplanung eine vom Gegenstromprinzip getragene „Raumordnung von unten“. Neben dem "soft law" maß Gatawis dem raumwirksamen Leistungsrecht in Form der Strukturfonds herausragende Bedeutung zu. Die Förderung erfolge hierbei durch spezielle Fonds für die regionale Ent- wicklung und die Landwirtschaft, durch Pilotprojekte und innovative Maßnahmen sowie durch Gemeinschaftsinitiativen wie Interreg, Urban und Leader. $\mathrm{Ga}$ tawis erläuterte, dass das EUREK und die Strukturfonds Zielkonformität aufwiesen und organisatorisch verknüpft seien. Die Effektivität des EUREK werde erhöht, indem seine Umsetzung als Bedingung für die Strukturförderung vorausgesetzt werde. Als dritte Säule des raumwirksamen Europarechts stellte Gatawis das Ordnungs- und Planungsrecht heraus. Hierbei ging er auf die Entscheidung zum Aufbau eines transeuropäischen Verkehrsnetzes, auf die Errichtung eines europäischen Schutzgebietssystems „Natura 2000“ gemäß der Vogelschutz- und der Flora-Fauna-Habitat-Richtlinie sowie auf die Richtlinie über die Umweltverträglichkeitsprüfung für Pläne und Programme ein.

Zurück auf die Landes- bzw. kommunale Ebene führte der Vortrag zum Thema „Dezentralisierung der Landesplanung" von Dr. Alexander Schink, Hauptgeschäftsführer des Landkreistages Nordrhein-Westfalen. Zunächst stellte Schink aus kommunaler Sicht dar, auf Grund welcher Probleme die Landesplanung dezentralisiert werden müsse. Er kritisierte die Starrheit des Verfahrens, die sich insbesondere im Genehmigungserfordernis für die Gebietsentwicklungsplanung äußere. Auch sei es nach bisherigem Verfahrensrecht nur unzureichend möglich, umweltrechtliche Prüfverfahren wie die Umweltverträglichkeitsprüfung, die Verträglichkeitsprüfung nach der Flora-Fauna-Habitat-Richtlinie sowie die naturschutzrechtliche Eingriffsregelung so in die Planungsebenen zu integrieren, dass eine Abschichtung möglich werde. Bisher würden in der nordrhein-westfälischen Landesplanung die Möglichkeiten und Anforderungen grenzüberschreitender Planungsverfahren nur ansatzweise berücksichtigt. Besonders betonte Schink, dass den Kommunen häufig bereits parzellenscharfe Vorgaben gemacht würden, so dass keine ausreichenden Gestaltungsspielräume verblieben. Daraus erwachse als Ziel, für eine Erneuerung der Landesplanung das Gegenstromprinzip nutzbar zu machen, indem den kommunalen Akteuren die Entscheidungen vor Ort überlassen werden sollten. Verbindliche landesplanerische Ziele sollten durch Leitbilder und Grundsätze ersetzt sowie flächenscharfe Aussagen vermieden werden. Die landesplanerischen Vorgaben könnten zudem auf das Wesentliche konzentriert werden, indem Landesplanungsgesetz, Landesentwicklungsprogramm und Landesentwicklungsplan in einem zusammenfassenden Planwerk aufgingen. Wie vor ihm schon Schneider wies auch Schink auf die Novellierungsbedürftigkeit des Verfahrensrechts hin. Er empfahl, die Genehmigungspflicht für Gebietsentwicklungspläne in eine Anzeigepflicht umzuwandeln, die nur bei Rechtsverstößen in 
ein Genehmigungsverfahren münden solle. In diesem Zusammenhang sei es von besonderer Wichtigkeit, die Kompetenzen der Regionalräte auszuweiten. Um durch Abschichtung eine effiziente Informationsverarbeitung und Kooperation der Planungsebenen zu erreichen, sprach Schink sich zudem für eine bessere Aufbereitung des Abwägungsmaterials aus. Er regte die Erarbeitung von Fachbeiträgen als Entscheidungsgrundlage im Verfahren zur Erstellung von Gebietsentwicklungsplänen an. Dadurch könnten Qualität und Akzeptanz der Planung gesteigert werden. Nicht nur um europarechtlichen Vorgaben gerecht zu werden, sei bei der Aufstellung von Gebietsentwicklungsplänen zudem eine Öffentlichkeitsbeteiligung geboten. Wie bei der hochstufigen Landesplanung seien auch bei der Gebietsentwicklungsplanung flächenbezogene, detaillierte Aussagen zu reduzieren. Bei naturschutzrechtlichen Festlegungen sei insofern zu differenzieren, als die Schutzgebiete des Systems "Natura 2000“ nach wie vor parzellenscharf ausgewiesen werden müssten, während für Ausgleichs- und Ersatzmaßnahmen nach der Eingriffsregelung Suchräume identifiziert und Flächenpools gesichert werden sollten. Um den Flächenverbrauch durch Siedlungen und Gewerbe zu kontrollieren, sollten Flächenobergrenzen und Tabuzonen festgesetzt werden. Auf der Ebene der Gebietsentwicklungsplanung solle nach Schink also vorrangig die Standortverträglichkeit geklärt werden. Zudem sollten kooperative Instrumente wie der landesplanerische Vertrag vermehrt zur Anwendung kommen.

Dieser letzte Aspekt wurde in einem weiteren Vortrag unter dem Titel „Verträge in der Landesplanung" von Sabine Klaßmann-Voß, Regierungsdirektorin in der Staatskanzlei des Landes Nordrhein-Westfalen, vertieft. Die Landesplanung stehe durch veränderte europäische und nationale Rahmenbedingungen vor neuen Aufgaben, die $z u$ einer intensiveren Zusammenarbeit zwischen allen Ebenen der Raumordnung herausforderten. Die klassischen Instrumente der Landesplanung seien dabei nicht flexibel genug und könnten sich gar entwicklungshindernd auswirken. Dem trage die Entwicklung der sog. „neuen“ Instrumente Rechnung, die Beweglichkeit gewährleisteten, indem sie das „bottom-up-Prinzip" verfolgten und auf einen Konsens unter den Beteiligten ausgerichtet seien. Unter diesen neuen Instrumenten gem. $\$ 13$ ROG sei insbesondere der landesplanerische Vertrag bedeutsam. Er erweitere die Gestaltungsmöglichkeiten der Planungsträger.
Klaßmann-Voß betonte jedoch, dass der landesplanerische Vertrag ein Planwerk nicht ersetzen, sondern einerseits zur Vorbereitung und andererseits zur Verwirklichung des Planes beitragen könne. Der Vertrag erhöhe die rechtliche Durchsetzungskraft der Landesplanung und wirke akzeptanzsteigernd. Klaßmann$V o ß$ erläuterte, dass es keinen gesetzlichen Katalog möglicher Inhalte wie im Baugesetzbuch gebe, so dass für den landesplanerischen Vertrag ein weiter Anwendungs- und Gestaltungsspielraum bestehe. Die Referentin stellte besonders die Möglichkeiten auf regionaler Ebene heraus und nannte als Beispiele die Umsetzung regionaler Entwicklungskonzepte und die interregionale Zusammenarbeit zur Entwicklung eines Gewerbegebietes. Die Rechtsnatur des landesplanerischen Vertrages richte sich nach dem Regelungsgegenstand; in der Regel handele es sich um einen öffentlichrechtlichen Vertrag, der seine rechtlichen Grenzen in $\$ \S 54$ ff. VwVfG finde. Zudem gelte das Verbot der Vorwegbindung. Wer Vertragspartner landesplanerischer Verträge werden könne, unterliege keinen gesetzlichen Beschränkungen. Die Motivation Privater werde durch finanzielle und faktische Vergünstigungen gefördert.

Die Vorträge und Diskussionen des Symposiums trugen dazu bei, die Probleme der heutigen Landesplanung zu konkretisieren, Erfahrungen auszutauschen sowie Lösungsansätze darzustellen und kritisch zu beleuchten. Die Novellierungsbedürftigkeit des Landesplanungsrechts wurde nicht lediglich ein weiteres Mal beklagt; vielmehr konnten Vorschläge gemacht und Lösungswege in eine erfolgversprechende „Zukunft der Landesplanung“ aufgezeigt werden.

\section{Anmerkungen}

* Ein ausführlicher Symposiumsbericht wird unter dem Titel „Zur Zukunft der Landesplanung - Neue Ansätze und Entwicklungen des Landesplanungsrechts“ als Band 203 der „Beiträge zur Raumplanung und zum Siedlungs- und Wohnungswesen“ in Münster erscheinen.

Dipl.-Landschaftsökol. Cornelia Wellens

Zentralinstitut für Raumplanung

an der Universität Münster

Wilmergasse $12-13$

48143 Münster 